\title{
An Experimental and Theoretical Investigation of a Micro Mirror under Mixed-Frequency Excitation
}

\author{
Saad Ilyas, Abdallah Ramini, Armando A. Carreñoda, and Mohammad I. Younis
}

\begin{abstract}
We present an experimental and theoretical investigation of a micro machined mirror under a mixed frequency signal composed of two harmonic AC sources. The micro mirror is made of Polyimide as the main structural layer.

The experimental and theoretical dynamics are explored via frequency sweeps in the desired neighborhoods. One frequency is fixed while the other frequency is swept through a wide range to study the dynamic responses. To simulate the behavior of the micro mirror, it is modeled as a single degree of freedom system, where the parameters of the model are extracted experimentally. Good agreement is reported among the simulation results and the experimental data. These responses are studied under different frequencies and input voltages. The results show interesting dynamics, where the system exhibits primary resonance, and combination resonances of additive and subtractive type. The mixed excitation is demonstrated as a way to increase the bandwidth of the resonator near primary resonance, which can be promising for resonant sensing applications in the effort to increase the signal-noise ratio over extended frequency range.
\end{abstract}

Index Terms-Micro Mirror, Mixed-frequency excitation, Multi-frequency excitation, Resonators, Electrostatic actuation, Polyimide, Bandwidth

\section{INTRODUCTION}

$\mathrm{E}$ XPLORING and exploiting the interesting dynamical behavior of MEMS is a widely studied area of research as most MEMS devices exhibit interesting dynamic behaviors introduced by either electrostatic actuation [1,2], stiffness [1] or damping [2]. It is necessary to understand these behaviors in order to either exploit them efficiently or avoid them, if needed. Considerable efforts have been directed recently to understand many of the complex dynamics phenomena at the micro scale including dynamics pull-in $[1,3]$ and parametric excitation [4-8]. These interesting phenomena also have been proposed for useful applications in mass sensing $[9,10]$, low activation voltage switches [11], and digital logic designs [12].

Recently the mixed frequency excitations of the micro and nano resonators have inspired great interest due to their exciting and motivating behaviors. These resonators have been proposed in applications in areas like spectroscopy [13, 14] because of their remarkable properties, where they have helped in calculating the refractive index of materials accurately and rapidly. Also, they were implemented in atomic force microscopy [15-21] to convey several levels of information about the sample under test, where every mode of vibration is responsible for a different kind of data (first mode for surface topology, second mode for charge distributions, etc). Another recent use of mixed-frequency excitation is in digital logic devices [12], where mixing of different frequencies result in distinct output frequencies, and hence enable logic operations. In addition, mixing of frequencies through quadratic electrostatic forces has been proposed in [22-27] to realize down converters, mixers, and filters. Mixed-frequency excitation has been used in NEMS resonators to avoid the feed through parasitic by generating a low frequency term [28]. In addition, it presents a way to detect a high frequency signal in NEMS by converting it into a low frequency signal using mixing without losing any information. Frequency modulation and digital demodulation of a carbon nanotube NEMS in transistor geometry is presented in [29]. A tunable carbon nanotube electromechanical oscillator using frequency mixing is presented in [30]. The identification and the characterization of bending vibrations of suspended semiconducting Single Walled Carbon Nano Tube resonators at room temperature, by using them as frequency mixers, is presented in [31].

The micro mirror is an exciting MEMS structure that has been widely used in optics [32, 33]. Also, seesaw type motion of a micro mirror has enabled the design of complementary universal MEMS logic devices [34, 35]. The dynamics of micro mirrors under mixed-frequency excitation has not yet been explored. The full potential and use of mixed frequency excitation and its exploitation for practical MEMS applications has not yet been investigated in depth, especially for torsional actuators and micro mirrors. Due to the interesting dynamics and exciting behavior of systems under this excitation it is necessary to exploit these dynamics in micro mirrors for sensing and actuations applications in MEMS. In this work, we investigate the dynamic behaviors of a micro mirror under mixed frequency excitations.

The organization of the paper is as follows. In Section 2 we discuss the design and fabrication process of the polyimide micro mirror based on surface micromachining techniques. Next, the mathematical model using lumped parameter techniques is discussed in Section 3. Section 4 presents the experimental setup and procedures used in gathering all the experimental data. Section 5 presents different case studies performed in order to understand the behavior of the system under mixed-frequency excitation. Next, Section 6 presents a discussion on the possible application and areas to be explored 
further. Finally, Section 7 summarizes the results and presents the conclusions.

\section{DESIGN AND FABRICATION}

\section{1) Design}

A micro mirror is basically a plate clamped by two flexure beams on both sides, which undergo torsion as the mirror actuates. The proposed micro mirror is a similar device as shown in the schematics in Fig.1.

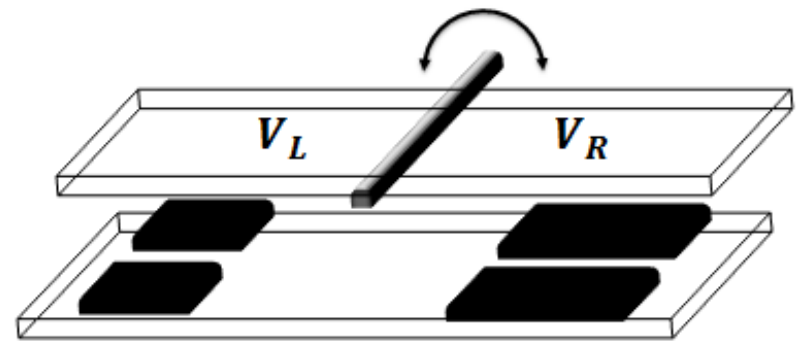

(a)

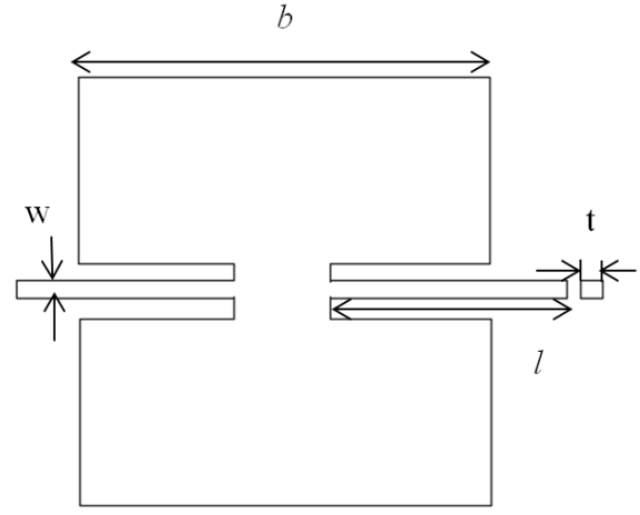

(b)

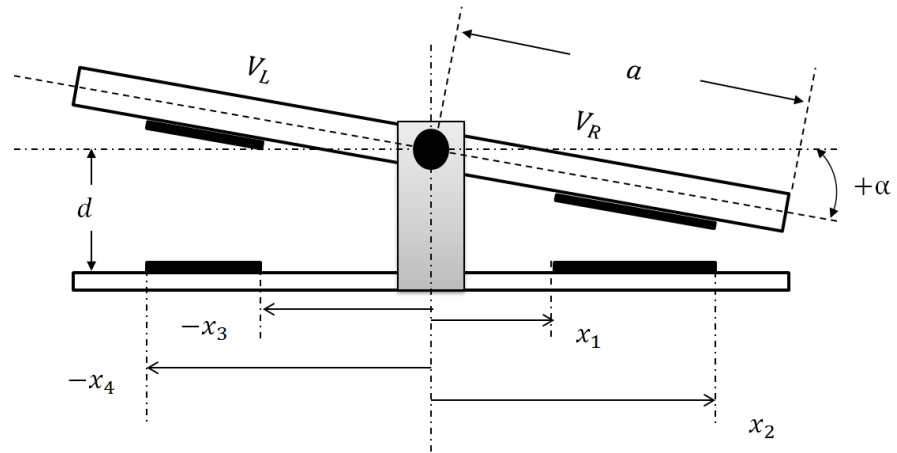

(c)

Fig. 1. Schematic views of the micro mirror (a) 3D view. (b) Top view. (c) 2D side view.

This mirror can be actuated in three different ways: The first way of actuation is through the right half of the mirror, which has larger fixed electrodes compared to the left side. The second way is through actuating the smaller electrodes on the left size. Finally, it can be actuated by one electrode of each side. Depending on the actuation type, various equilibrium positions of the mirror and its natural frequency can be set. Fig. 2 shows a simulated plot of frequency against actuation voltage for the three methods of actuation. It can be noticed how at a single fixed voltage the device can be actuated at three different frequencies. Alternatively, a single fixed frequency of the device can be activated using three different voltages depending on how the device is excited. This method can prove useful in resonator applications as it provides three different control points. We can choose to operate around any one depending on the actuation method. This paper considers the case of electrostatic actuation through the right half of the mirror, which has the larger fixed electrodes.

Table 1 summarizes the measured dimensions and other important parameters of the system under consideration.

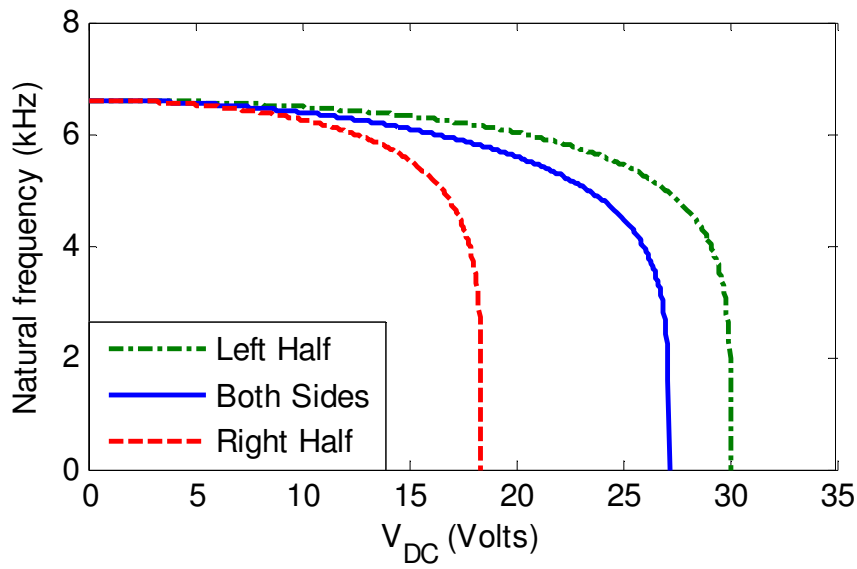

Fig. 2. Variation of the fundamental natural frequency against voltage under three methods of actuation (Left Half: smaller electrodes, Right half: larger electrodes, Both sides: one electrode on each side).

TABLE 1

SPECIFICATIONS OF THE MICRO MIRROR

Length of the device
Width of the device

Electrode location

Electrode location

Electrode location

Electrode location

Smaller electrode thickness

\begin{tabular}{l|r}
$2 a$ & $535 \mu \mathrm{m}$ \\
$b$ & $150 \mu \mathrm{m}$
\end{tabular}

Larger electrodes thickness

Flexure beam length

Flexure beam width

Flexure beam thickness

Gap

Maximum tilt angle of mirror

$\begin{array}{lll}x_{1} & 97.5 \mu \mathrm{m}\end{array}$

Pull in voltage for right half

$222.5 \mu \mathrm{m}$

$-105 \mu \mathrm{m}$

$-185 \mu \mathrm{m}$

$30 \mu \mathrm{m}$

$42.5 \mu \mathrm{m}$

$100 \mu \mathrm{m}$

$15 \mu \mathrm{m}$

$5 \mu \mathrm{m}$

$5 \mu \mathrm{m}$

$4.38 \mathrm{mrad}$

18.4 volts

\section{2) Fabrication}

The micro mirror is fabricated using a 6 layer fabrication process based on surface micromachining techniques. Fig. 3 shows the fabrication cross section of the right half of the mirror. It is approximately $7 \mu \mathrm{m}$ thick in total comprising of a polyimide structural layer with a nominal thickness of $6 \mu \mathrm{m}$. Also, it is separated by a $5 \mu \mathrm{m}$ gap from its bottom electrodes, which are patterned on the Si substrate. Metal_0 layer of $50 \mathrm{~nm} / 250 \mathrm{~nm}$ of $\mathrm{Cr} / \mathrm{Au}$ forms these fixed ground electrodes. A $50 \mathrm{~nm} / 250 \mathrm{~nm} / 50 \mathrm{~nm}$ layer of $\mathrm{Cr} / \mathrm{Au} / \mathrm{Cr}$ forms the Metal_1 layer patterned onto the structural layer forming the movable gate electrodes. Anchors are used to hold the actuator to the ground as well as to provide electrical signal to the moveable gate electrodes whereas dimples are incorporated to avoid the electrical shorting of the electrodes upon pull in. Metal_2 layer, as shown in Fig. 3, comprises of 450nm of Ni patterned similar 
to Metal_1 layer to avoid the bimorph effect and to have a straight structure.

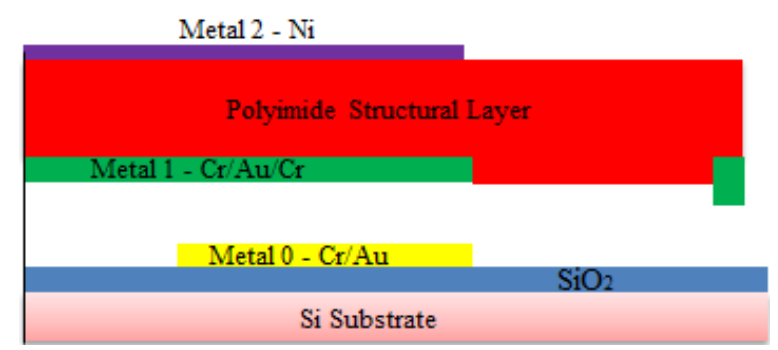

Fig. 3. Fabrication process cross-section of one side of the mirror.

Fig. 4 shows the SEM image of the fabricated micro mirror. The image shows two anchors holding the mirror through flexure beams and allowing its torsional motion. The patterned metal visible on top of the polyimide structural layer is Ni used to counter for the bimorph effect.

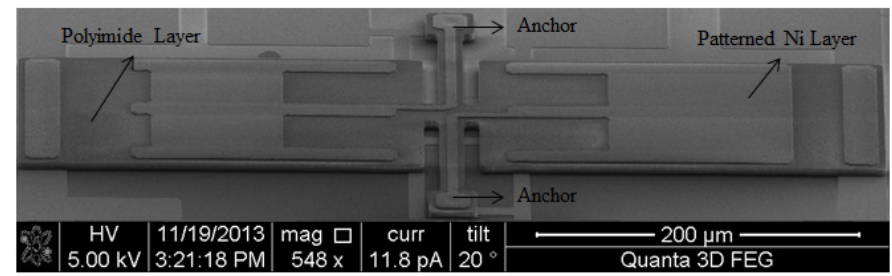

Fig. 4. A SEM image of the fabricated micro mirror.

\section{MODELING}

The mirror is modeled as a single degree of freedom system [36]. The equation of motion can be expressed as

$$
I \ddot{\alpha}+c \dot{\alpha}+K_{t} \alpha=M_{R}-M_{L}
$$

Where, $I$ is the mass moment of inertia, $c$ is the damping constant, $K_{t}$ is the torsional stiffness, $M_{L}$ is moment on the left side of mirror, and $M_{R}$ is the moment on the right side of the mirror, which can be expressed as

$$
M_{R}=\frac{\varepsilon b_{r} V_{R}}{2 \alpha^{2}}\left[\frac{d}{\left(d-x_{2} \alpha\right)}-\frac{d}{\left(d-x_{1} \alpha\right)}+\ln \left\{\frac{\left(d-x_{2} \alpha\right)}{\left(d-x_{1} \alpha\right)}\right\}\right]
$$

Where, $\varepsilon$ is the dielectric constant of gap medium, $V_{R}$ is the potential difference on the right side of mirror and $\alpha$ is the tilt angle of the mirror. Similarly

$$
M_{L}=\frac{\varepsilon b_{l} V_{L}}{2 \alpha^{2}}\left[\frac{d}{\left(d-x_{4} \alpha\right)}-\frac{d}{\left(d-x_{3} \alpha\right)}+\ln \left\{\frac{\left(d-x_{4} \alpha\right)}{\left(d-x_{3} \alpha\right)}\right\}\right]
$$

Where $V_{L}$ is the potential difference on the left side of mirror, which can be expressed as

$$
V_{L}=V_{R}=\left[V_{D C}+V_{A C 1} \cos \left(\Omega_{1}\right)+V_{A C 2} \cos \left(\Omega_{2}\right)\right]^{2}
$$

Where, $V_{A C l}$ is the amplitude of the first AC source, $V_{A C 2}$ is the amplitude of the second AC source, $V_{D C}$ is the polarization voltage and $\Omega_{1}, \Omega_{2}$ are the first and second excitation frequencies, respectively.

The torsional stiffness $K_{t}$ of the flexures can be calculated as

$$
K_{t}=\frac{2 G J_{P}}{l}
$$

Where $G$ is the shear modulus and $J_{P}$ is the polar moment of inertia. The parameter $J_{P}$ can be calculated as [37]

$$
J_{P}=\frac{1}{3} w t^{3}\left(1-\frac{192 t}{\pi^{5} w} \sum_{0}^{\infty} \frac{1}{[2 n+1]^{5}} \tanh \left[\frac{(2 n+1) \pi w}{2 t}\right]\right)
$$

Next we introduce the following normalized parameters:

$$
\begin{gathered}
\theta=\frac{\alpha}{\alpha_{\max }} \\
\alpha_{\max }=\frac{d}{a} \\
\gamma_{1}=\frac{x_{1}}{a} ; \gamma_{2}=\frac{x_{3}}{a} \\
\beta_{1}=\frac{x_{2}}{a} ; \beta_{2}=\frac{x_{4}}{a} \\
\hat{t}=\frac{t}{T} ; \quad \mu=\frac{c T}{I} ; \quad T=\sqrt{\frac{I}{K_{t}}}
\end{gathered}
$$

Using (7-11), the final normalized equation is written as

$$
\begin{aligned}
& \ddot{\theta}+\mu \dot{\theta}+\theta=\frac{\eta_{1}}{\theta^{2}}\left[\frac{1}{\left(1-\beta_{1} \theta\right)}-\frac{1}{\left(1-\gamma_{1} \theta\right)}+\ln \left(\frac{\left(1-\beta_{1} \theta\right)}{\left(1-\gamma_{1} \theta\right)}\right)\right] \\
& -\frac{\eta_{2}}{\theta^{2}}\left[\frac{1}{\left(1-\beta_{2} \theta\right)}-\frac{1}{\left(1-\gamma_{2} \theta\right)}+\ln \left(\frac{\left(1-\beta_{2} \theta\right)}{\left(1-\gamma_{2} \theta\right)}\right)\right]
\end{aligned}
$$

Where $\theta$ is the normalized tilt angle and

$$
\eta_{1}=\frac{\varepsilon b_{r} V_{R}}{2 \alpha_{\max }^{3} K_{t}} \quad ; \quad \eta_{2}=\frac{\varepsilon b_{l} V_{L}}{2 \alpha_{\max }^{3} K_{t}}
$$

\section{EXPERIMENTAL SETUP}

Fig. 5(a) shows a schematic of the experimental setup. The micro mirror is placed inside the vacuum chamber with a laser coming from a Laser Doppler Vibrometer (LDV) pointing directly at it to take the measurements. The LDV generates all the experimental results as displacement, which is then converted into tilt angle by relating the vertical displacement of the mirror with length span of half of the mirror. A data acquisition card, (DAQ) NI 6251from National Instruments, is used to acquire the data from the LDV. A resistor is installed to limit the current passing through the circuit in the event of pull in. It is worth mentioning that when driving this mirror harmonically by capacitive forces, there might be very small current generated in the current-limiting resistor due to the time varying capacitor. However, this is negligible compared to the current that would pass in the case of a pull-in (short circuit), which is the intended purpose of the resistor (i.e., to protect the mirror when pulled-in and when passing a current across it.) 

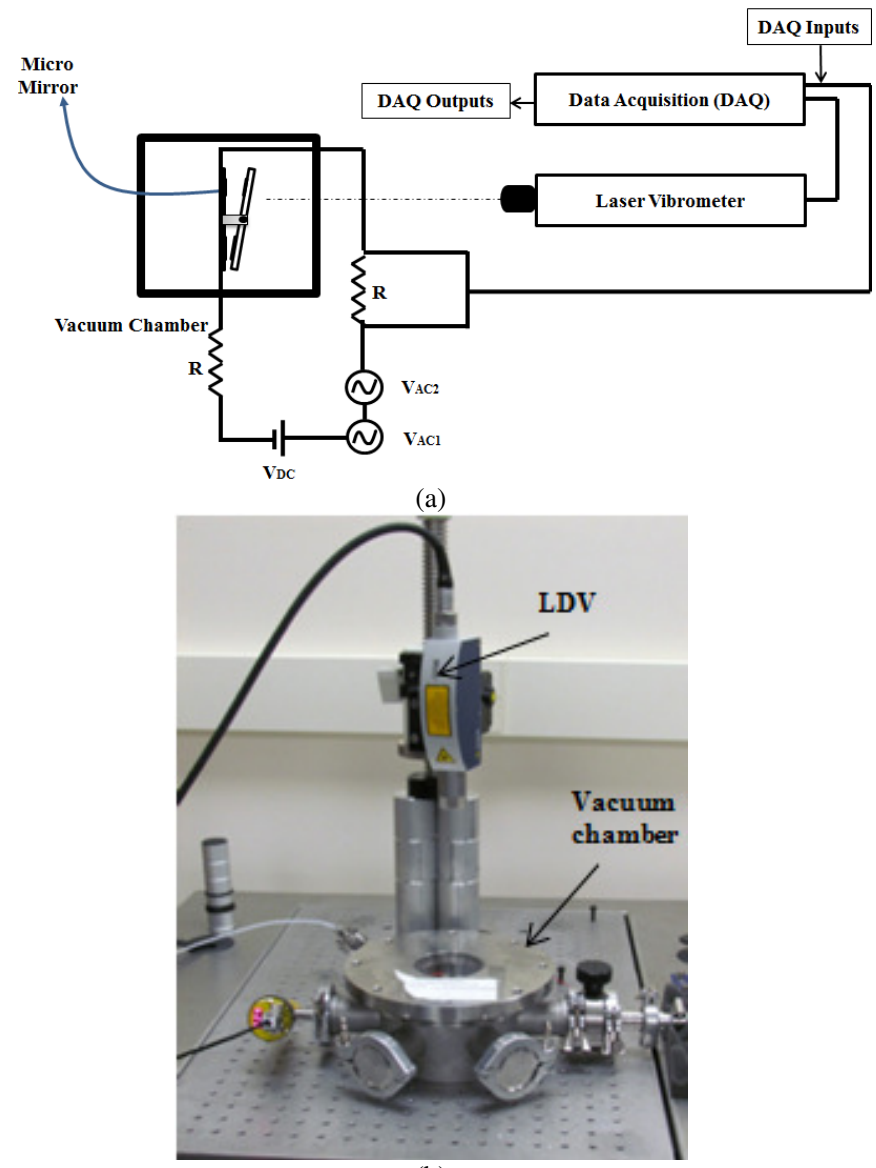

(b)

Fig. 5. (a) Schematic of the experimental setup. (b) LDV pointing at the micro mirror inside the vacuum chamber.

\section{MIXED-FREQUENCY EXCITATION RESUltS}

Next, we demonstrate and show results for the mirror under mixed-frequency excitation signals. The combination resonances look similar to what has been reported in $[38,39]$ due to the presence of quadratic nonlinearity. However, in the present case, these resonances are activated due to both effects of quadratic nonlinearities, due to the electrostatic force, and due to the quadratic form of the voltage in the numerator of the electrostatic force expression. To better understand this, one can expand the quadratic voltage term in (4), which yields

$$
\begin{aligned}
& V_{R}=V_{L}=V_{D C}{ }^{2}+V_{A C 1}{ }^{2} \cdot \cos ^{2}\left(\Omega_{1} t\right)+V_{A C 2}{ }^{2} \cdot \cos ^{2}\left(\Omega_{2} t\right) \\
& +2 V_{D C} \cdot V_{A C 1} \cdot \cos \left(\Omega_{1} t\right)+2 V_{D C} \cdot V_{A C 2} \cdot \cos \left(\Omega_{2} t\right) \\
& +V_{A C 1} \cdot V_{A C 2} \cdot\left[\cos \left\{\left(\Omega_{1}-\Omega_{2}\right) t\right\}+\cos \left\{\left(\Omega_{1}+\Omega_{2}\right) t\right\}\right]
\end{aligned}
$$

It is clear from (14) that the last term is responsible for producing the apparent resonances of additive and subtractive type. An additive type resonance will appear when the sum of the fixed frequency $\left(\Omega_{1}\right)$ and variable frequency $\left(\Omega_{2}\right)$ equals the natural frequency $\left(\omega_{n}\right)$ of the system i.e. $\Omega_{1}+\Omega_{2}=\omega_{n}$, whereas a subtractive type resonance appears when the difference of the fixed frequency and variable frequency equals the natural frequency of the system i.e. $\Omega_{1}-\Omega_{2}=\omega_{n}$. Note that in this paper we assume $\Omega_{1}$ to be the fixed frequency and $\Omega_{2}$ to be the variable frequency for all of the experimental results.

\section{A. Results and Discussions}

The primary natural frequency of the system is calculated using the finite element software ANSYS [40]. These results are obtained by basic modal analysis over the geometry of the micro mirror meshed using 3-D solid brick elements. The primary resonance frequency is calculated to be at $6.62 \mathrm{kHz}$. Fig. 6 shows the experimentally measured and theoretically calculated primary natural frequency of the system, which is consistent with that of the finite element calculation. The $y$ axis scale shows the displacement measurement obtained from the laser vibrometer as well as the tilt angle $\alpha$ calculated from relating the measured displacement to the length span of one side of the mirror. From now onwards all the plots will be shown against the tilt angle $\alpha$ as it relates more closely to the motion of the micro mirror.

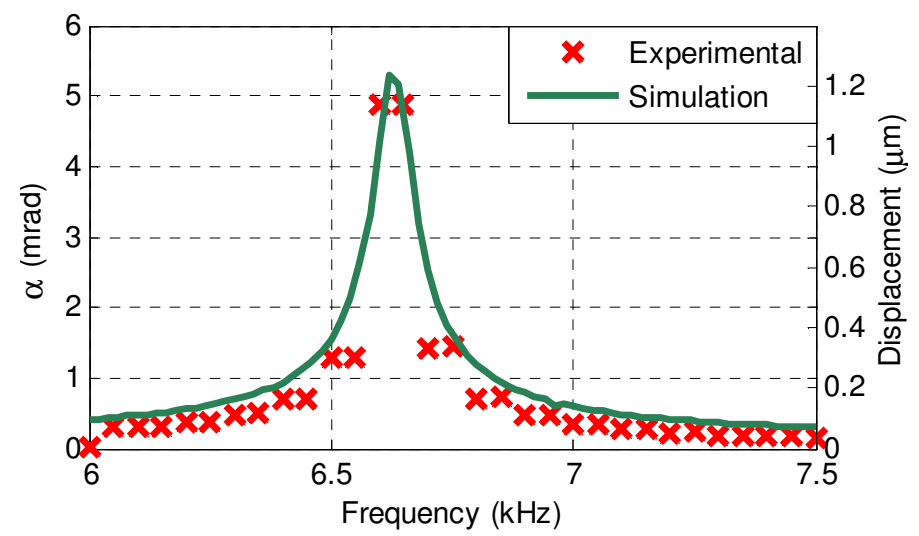

Fig. 6. Frequency response sweep at $V_{D C}=3 \mathrm{~V}, V_{A C I}=1 \mathrm{~V}$ to estimate the natural frequency of the mirror (around $6.62 \mathrm{kHz}$ ).

The method of extracting the damping coefficient $\xi$, the gap $d$, and the stiffness $K_{t}$ is explained in details in [41]. The effective mass moment of inertia $I$ is then calculated by using the results of the experimentally calculated natural frequency value $\omega_{n}$ and torsional stiffness $K_{t}$ in the below equation

$$
I=\frac{K_{t}}{\omega_{n}^{2}}
$$

The extracted parameters of the device are given in Table 2 .

TABLE 2

EXTRACTED PARAMETERS OF THE MICRO MIRROR

\begin{tabular}{ll|l}
\hline Torsional stiffness of the device & $K_{t}$ & $3.2 \mathrm{e}^{-8} \mathrm{~N} / \mathrm{m}$ \\
Effective mass moment of inertia & $I$ & $1.8082 \mathrm{e}-17 \mathrm{kgm}^{2}$ \\
Damping ratio & $\xi$ & 0.02 \\
\hline
\end{tabular}

Next, we show the results for various loading cases, where we fix one of the $\mathrm{AC}$ frequencies and vary the other one in the neighborhood of primary resonance. The effects of varying the fixed frequency and the applied voltage on the combination resonances are studied in these cases. The simulation part of the results is obtained by numerically integrating (12) in time using Runge-Kutta method [40]. All the results are post-processed and presented in the form of frequency response plots. 
1) Case 1: $V_{D C}=2 \mathrm{~V}, V_{A C l}=2 \mathrm{~V}, V_{A C 2}=1 \mathrm{~V}$, and $\Omega_{1}=500 \mathrm{~Hz}$

Initially, we consider the case when $\Omega_{1}$ is equal $500 \mathrm{~Hz}$ and $\Omega_{2}$ is swept over $2 \mathrm{kHz}$ frequency range around the primary resonance. One can note the resulting frequency response curve in Fig. 7. The figure shows the resonances of subtractive type at $7.1 \mathrm{kHz}$, due to the $\Omega_{1}-\Omega_{2}$ term in (14), and the additive type at $6.1 \mathrm{kHz}$, due to the $\Omega_{1}+\Omega_{2}$ term in (14), as expected. It can also be observed that the model accurately predicts these combination resonances and the amplitude of the peaks are also in good agreement. The deviation between theory and experiment can be attributed to the fact that we use lumped-parameter model for the distributed-parameter system (beams and plate).

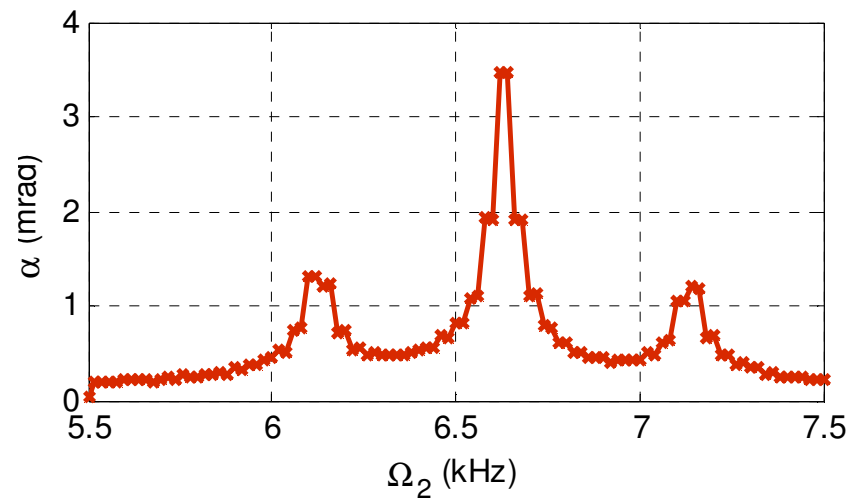

(a)

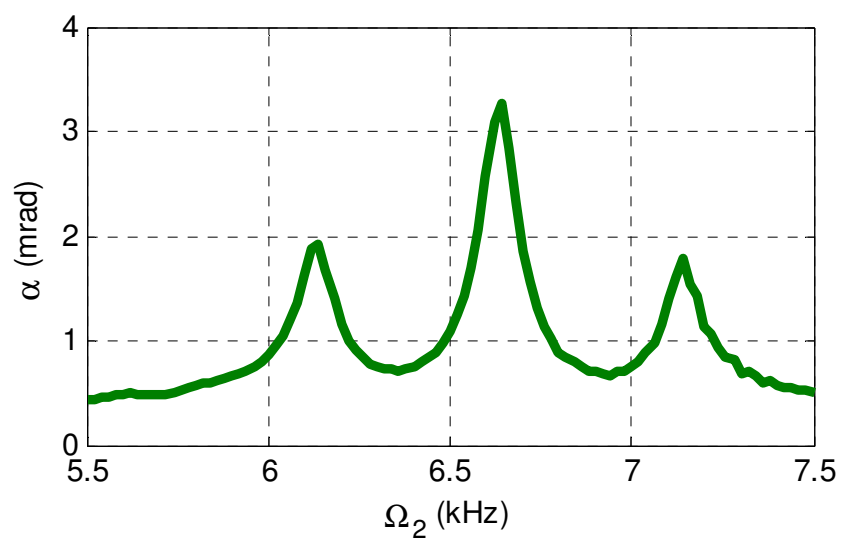

(b)

Fig. 7. (a) Measured frequency response sweep for $V_{D C}=2 \mathrm{~V}, V_{A C l}=2 \mathrm{~V}$, $V_{A C 2}=1 \mathrm{~V}, \Omega_{1}=500 \mathrm{~Hz}$. (b) Simulated frequency response sweep for $V_{D C}=2 \mathrm{~V}$, $V_{A C l}=2 \mathrm{~V}, V_{A C 2}=1 \mathrm{~V}, \Omega_{1}=500 \mathrm{~Hz}$.

2) Case 2: $V_{D C}=2 \mathrm{~V}, V_{A C l}=5 \mathrm{~V}, V_{A C 2}=1 \mathrm{~V}$ and $\Omega_{1}=2 \mathrm{kHz}$

Next, we consider another case just to further demonstrate that the shifting of the additive and subtractive resonances at the desired frequencies is controlled by the fixed frequency through the mixing. Fig. 8 demonstrates the experimental result for the additive type resonance, when $\Omega_{1}$ is fixed at $2 \mathrm{kHz}$. The resonances here are expected at $4.6 \mathrm{kHz}$ (additive type) and 8.6 $\mathrm{kHz}$ (subtractive type).

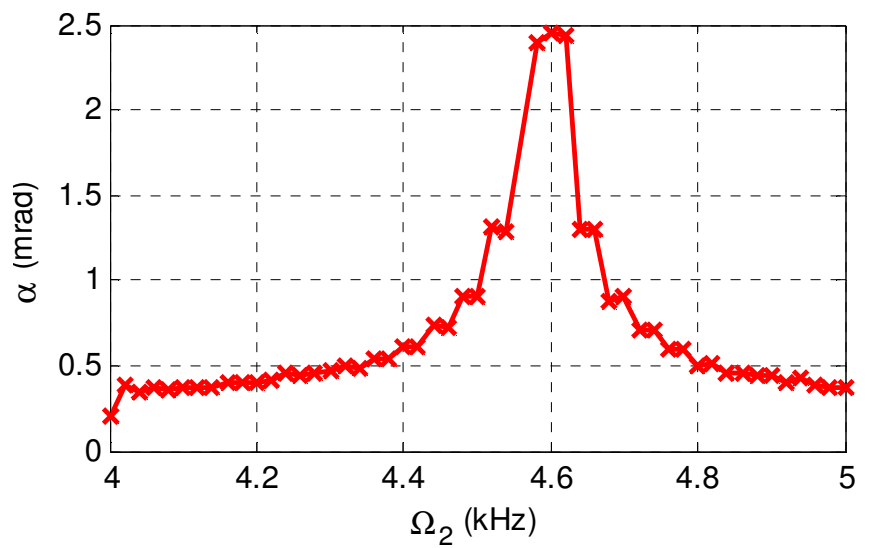

(a)

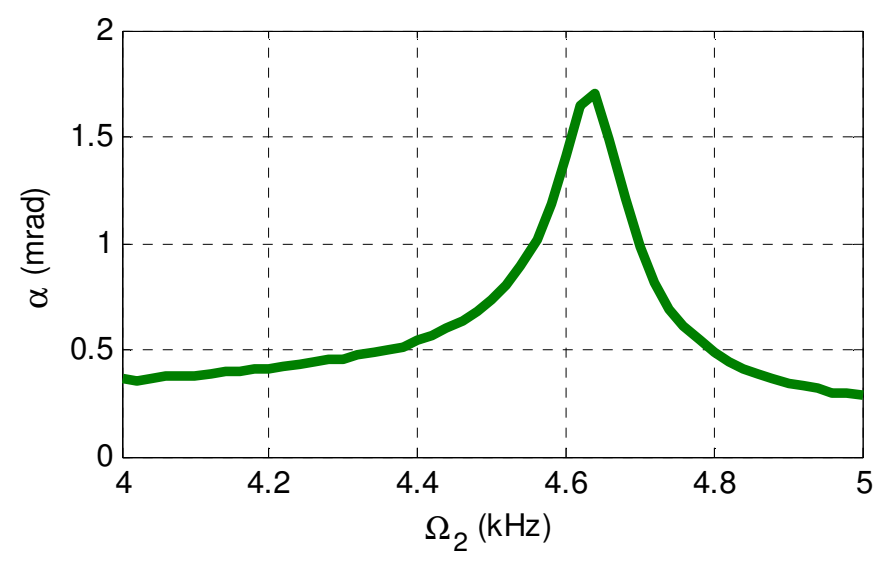

(b)

Fig. 8. (a) Measured frequency response sweep for $V_{D C}=2 \mathrm{~V}, V_{A C I}=5 \mathrm{~V}$, $V_{A C 2}=1 \mathrm{~V}, \Omega_{1}=2 \mathrm{kHz}$. (b) Simulated frequency response sweep for $V_{D C}=2 \mathrm{~V}$, $V_{A C l}=5 \mathrm{~V}, V_{A C 2}=1 \mathrm{~V}, \Omega_{1}=2 \mathrm{kHz}$.

3) Case 3: $V_{D C}=2 \mathrm{~V}, V_{A C l}=5 \mathrm{~V}, V_{A C 2}=1 \mathrm{~V}$, and $\Omega_{1}=100 \mathrm{kHz}$

In this case, we study the behavior of these resonances at a very high fixed frequency compared to the natural frequency of the mirror i.e. $100 \mathrm{kHz}$. Fig. 9 compares the experimental data of this case with and without mixed-frequency excitation. Resonance is activated at $106.6 \mathrm{kHz}$ due to the $\Omega_{1}-\Omega_{2}$ condition of the combination resonances in case of mixed frequency-excitation while no response from the system is observed in case of only single frequency excitation. Using this technique, we can shift any one of the additive or subtractive type resonances to any desired frequency. Also a huge difference in amplitude can be observed between the single source and mixed-frequency excitation, which shows the effectiveness of this method. A similar peak (not shown) is also activated at $94.4 \mathrm{kHz}$, again due to the $\Omega_{1}-\Omega_{2}$ term (100-94.4 $=$ $6.6 \mathrm{kHz}$ ), which is essentially a subtractive type. Hence, resonance is observed at $\Omega_{2}=94.4 \mathrm{kHz}$. 


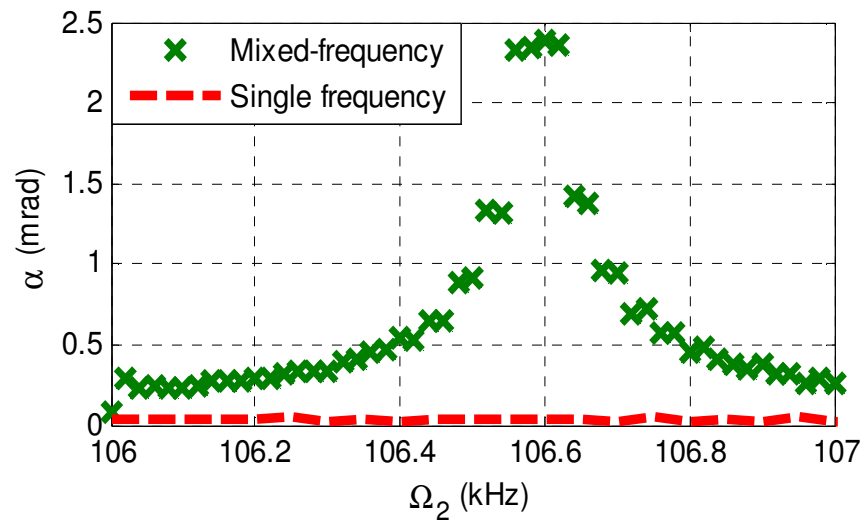

Fig. 9. Measured frequency response sweep for $V_{D C}=2 \mathrm{~V}, V_{A C l}=5 \mathrm{~V}$, $V_{A C 2}=1 \mathrm{~V}, \Omega_{1}=100 \mathrm{kHz}$.

4) Case 4: $V_{D C}=2 \mathrm{~V}, V_{A C l}=$ variable, $V_{A C 2}=1 \mathrm{~V}, \Omega_{1}=500 \mathrm{~Hz}$

It is also clear from (14) that if the voltage $\left(V_{A C l}\right)$ associated with the fixed frequency $\left(\Omega_{1}\right)$ is increased and the rest of the parameters are kept the same, it results in higher amplitude of the combination resonances. Fig. 10 demonstrates this effect from the experimental data obtained for different values of $V_{A C l}$. It shows how the amplitude increases from $1.2 \mathrm{mrad}$ to $3.2 \mathrm{mrad}$ until it is almost equal to the amplitude at primary natural frequency. Also, an increase in the amplitude of regions between the resonances can also be observed. This can effectively increase the bandwidth of the resonator, using the mixed-frequency excitation. In another words, mixed-frequency excitation can be proposed as an effective way to excite resonance sensors to achieve large signal-noise ratio over extended range of frequency, and thus avoiding the problem of a narrow sharp response over a limited range of frequency, which negatively affects the performance of devices, such as MEMS gyroscopes.

The results of the above discussed cases show that resonances can be activated at any frequency with the desired amplitude as long as we properly choose the input voltages. The ability to generate multiple resonance peaks and the ability to control how close they can be activated to each other as well as their amplitude without changing the geometry of the device is very promising feature that can be widely used in many MEMS applications, such as resonant sensors and other applications.

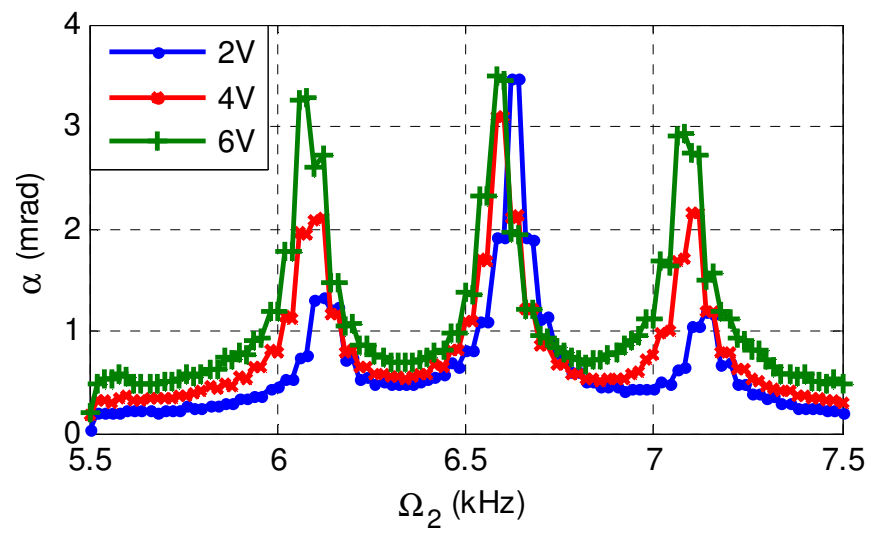

Fig. 10. Measured frequency response sweep for $V_{D C}=2 \mathrm{~V}, V_{A C l}=$ variable, $V_{A C 2}=1 \mathrm{~V}, \Omega_{1}=500 \mathrm{~Hz}$.

\section{DISCUSSION}

\section{A. Resonators Applications}

As indicated earlier, the mixed-frequency excitation can effectively increase the bandwidth of resonators. This occurs when the combination resonances (additive and subtractive) get closer to the primary natural frequency as the fixed excitation frequency gets small. This results in increasing the bandwidth around the primary resonance. Fig. 11 shows the experimental results of reducing the fixed frequency to small values and its effect on the bandwidth of the micro mirror. In this figure, we increase the bandwidth around the primary natural frequency. Also, a very high amplitude of the combination resonances can be achieved by selecting the proper voltage input. This proves that using mixed-frequency excitation can increase the bandwidth in resonators without making any changes to the device design or sacrificing much of its maximum amplitude. This can be advantageous in applications, such as MEMS gyroscopes, where mismatch problems can result in a huge loss in the signals [43].

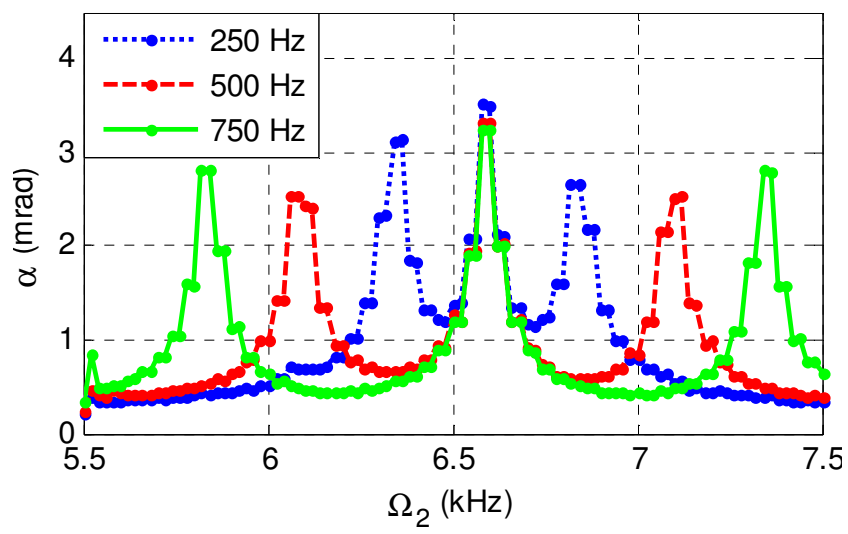

Fig. 11. Measured frequency response sweep for $V_{D C}=2 \mathrm{~V}, V_{A C l}=5 \mathrm{~V}$, $V_{A C 2}=1 \mathrm{~V}, \Omega_{1}=$ variable.

The bandwidth can be further increased if more than two sources of excitation are applied, since each new frequency gives rise to two new resonance peaks around the primary resonance. Fig. 12 shows simulation results of a case where the mirror is excited with four $\mathrm{AC}$ frequency sources. It can be observed that there are six resonances surrounding the primary resonance; each associated with the respective fixed frequencies, which in this case are $100 \mathrm{~Hz}, 200 \mathrm{~Hz}$ and $350 \mathrm{~Hz}$. It can be observed that the bandwidth of resonators can be effectively increased without introducing any complexity in the design or fabrication.

\section{B. Energy Harvester Applications}

Contrary to the previous case, where the fixed frequency was given a value close to zero, if the magnitude of the fixed frequency is set very close to the primary natural frequency, the additive type resonance can be activated at a very small frequency range (close to $0 \mathrm{~Hz}$ ). Fig. 13 shows the effect of setting the fixed frequency very close to the primary natural frequency based on experimental data. It can be observed that very high amplitude peaks are activated between 100-300 Hz depending upon the fixed frequency value. However, we 
cannot observe anything in the case of a single source excitation $\left(V_{A C l}=0\right)$. Also in this figure, the amplitude of the sub resonance is very high and is spread across a wide range of frequencies when the fixed frequency is very close to the natural frequency i.e. $6.4 \mathrm{kHz}$. This can be promising for energy harvesting; since it provides the system with resonances of very high amplitudes at very low frequency ranges regardless of the natural frequency of the system. It also allows targeting any frequency range where these energy harvesters are desired to operate, which otherwise is possible only by changing the design and refabricating a new device. Of course, the energy efficiency of adding an external source at a fixed frequency to activate the combination resonance needs to be investigated in details before judging on the suitability of this method and its feasibility.

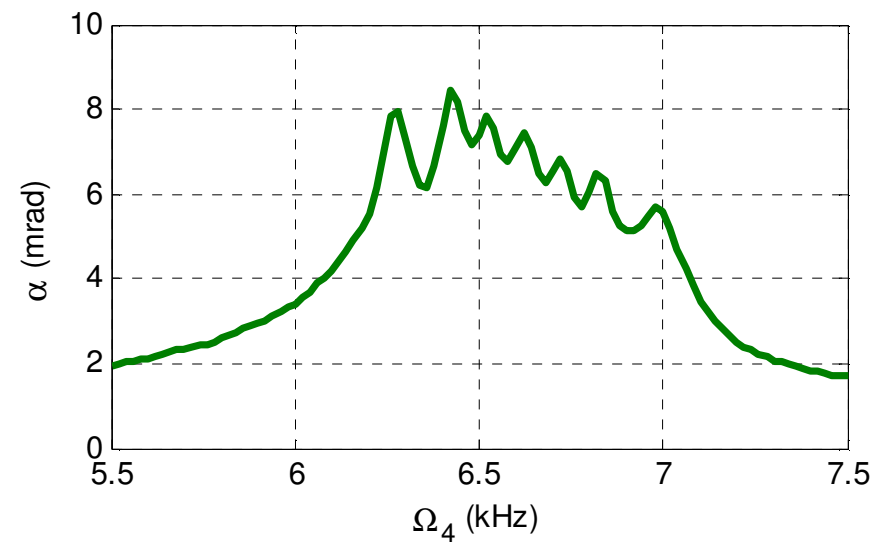

Fig. 12. Simulated frequency sweep for $V_{D C}=1.5 \mathrm{~V}, V_{A C l}=1.85 \mathrm{~V}, V_{A C 2}=1.85 \mathrm{~V}$ $V_{A C 3}=2.5 \mathrm{~V}, V_{A C 4}=2.5 \mathrm{~V}, \Omega_{1}=100 \mathrm{~Hz}, \Omega_{2}=200 \mathrm{~Hz}$, and $\Omega_{3}=350 \mathrm{~Hz}$.

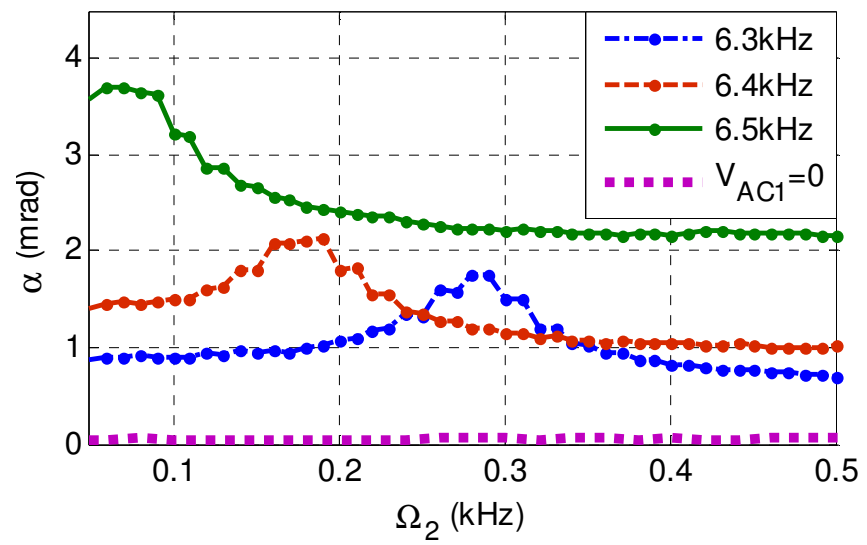

Fig. 13. Measured frequency sweep for $V_{D C}=2 \mathrm{~V}, V_{A C l}=5 \mathrm{~V}, V_{A C 2}=1 \mathrm{~V}, \Omega_{1}=$ variable.

\section{CONCLUSIONS}

Multi and mixed-frequency excitation has been studied both theoretically and experimentally based on a Polyimide micro-mirror. The mixed frequency excitation can be effectively used in resonators and resonant sensors to increase their bandwidths, by pushing the combination resonances close to their primary natural frequencies and by maintaining the overall amplitude in the vicinity of the primary natural frequency. Furthermore, it is possible to implement this method for energy harvesters to force the combination resonances to small range of few hundred Hertz and to maintain very high amplitude at that range. All of this is enabled due to the precise control over the amplitude and frequencies of these combination resonances, provided by the mixed-frequency excitation.

\section{ACKNOWLEDGMENT}

This work has been supported through King Abdullah University of Science and Technology (KAUST) research funds.

\section{REFERENCES}

[1] A. H. Nayfeh, M. I. Younis, and E. M. Abdel-Rahman, "Dynamic pull-in phenomenon in MEMS resonators," Nonlinear. Dynam., vol. 48, pp. 153-163, 2007.

[2] P. Li, R. Hu, and Y. Fang, "A new model for squeeze-film damping of electrically actuated microbeams under the effect of a static deflection," $J$. Micromech Microeng, vol. 17, p. 1242, 2007.

[3] S. Krylov and R. Maimon, "Pull-in dynamics of an elastic beam actuated by continuously distributed electrostatic force," J.Vib. Acoust, vol. 126, pp. 332-342, 2004.

[4] H. Yamaguchi, H. Okamoto, and I. Mahboob, "Coherent control of micro/nanomechanical oscillation using parametric mode mixing," App. Phys. Exp., vol. 5, p. 014001, 2012.

[5] I. Mahboob, V. Nier, K. Nishiguchi, A. Fujiwara, and H. Yamaguchi, "Multi-mode parametric coupling in an electromechanical resonator," App.l Phys. Lett, vol. 103, p. 153105, 2013.

[6] H. Yamaguchi and I. Mahboob, "Parametric mode mixing in asymmetric doubly clamped beam resonators," New J. Phys, vol. 15, p. 015023, 2013.

[7] I. Mahboob and H. Yamaguchi, "Piezoelectrically pumped parametric amplification and Q enhancement in an electromechanical oscillator," Appl Phys Lett, vol. 92, p. 173109, 2008.

[8] I. Mahboob, M. Mounaix, K. Nishiguchi, A. Fujiwara, and H. Yamaguchi, "A multimode electromechanical parametric resonator array," Scientific reports, vol. 4, 2014.

[9] M. I. Younis and F. Alsaleem, "Exploration of new concepts for mass detection in electrostatically-actuated structures based on nonlinear phenomena," J. Comput Nonlinear Dynam., vol. 4, p. 021010, 2009.

[10] W. Zhang and K. L. Turner, (2004), "A mass sensor based on parametric resonance," Proc. of the Solid State Sensor, Actuator and Microsystem Workshop, Hilton Head Island, SC, pp. 49-52.

[11] A. Fargas-Marques, J. Casals-Terré, and A. M. Shkel, "Resonant pull-in condition in parallel-plate electrostatic actuators," J. Microelectromech Syst, S, vol. 16, pp. 1044-1053, 2007.

[12] I. Mahboob, E. Flurin, K. Nishiguchi, A. Fujiwara, and H. Yamaguchi, "Interconnect-free parallel logic circuits in a single mechanical resonator," Nature comm., vol. 2, p. 198, 2011.

[13] R. Adair, L. Chase, and S. A. Payne, "Nonlinear refractive-index measurements of glasses using three-wave frequency mixing," JOSA B, vol. 4, pp. 875-881, 1987.

[14] M. Levenson, "Feasibility of measuring the nonlinear index of refraction by third-order frequency mixing," Quant Electron, 1974.

[15] R. Garcia and E. T. Herruzo, "The emergence of multifrequency force microscopy," Nature nanotech, vol. 7, pp. 217-226, 2012.

[16] D. Forchheimer, D. Platz, E. A. Tholén, and D. B. Haviland, "Model-based extraction of material properties in multifrequency atomic force microscopy," Phys. Rev. B, vol. 85, p. 195449, 2012.

[17] S. Hornstein and O. Gottlieb, "Nonlinear multimode dynamics and internal resonances of the scan process in noncontacting atomic force microscopy," J. Appl. Phys, vol. 112, pp. -, 2012.

[18] H. Westra, H. van der Zant, and W. Venstra, "Modal interactions of flexural and torsional vibrations in a microcantilever," Ultramicroscopy, 
vol. 120, pp. 41-47, 2012.

[19] M. G. Ruppert, M. W. Fairbairn, and S. Moheimani,( 2013) "Multi-mode resonant control of a microcantilever for Atomic Force Microscopy," in IEEE/ASME Int. Adv. Intelligent Mech. (AIM), pp. 77-82.

[20] K. Karvinen and S. Moheimani, "Control of the higher eigenmodes of a microcantilever: Applications in atomic force microscopy," Ultramicroscopy, vol. 137, pp. 66-71, 2014.

[21] A. Sebastian, N. Shamsudhin, H. Rothuizen, U. Drechsler, W. W. Koelmans, H. Bhaskaran, et al., "Note: Micro-cantilevers with AlN actuators and PtSi tips for multi-frequency atomic force microscopy," Rev. Scientific Ins, vol. 83, p. 096107, 2012.

[22] A. Erbe and R. H. Blick, "Silicon-on-insulator based nanoresonators for mechanical mixing at radio frequencies," IEEE T. Ultrason Ferr, vol. 49, pp. 1114-1117, 2002

[23] S. Pourkamali, R. Abdolvand, G. K. Ho, and F. Ayazi, "Electrostatically coupled micromechanical beam filters, IEEE MEMS, (2004) pp. 584-587.

[24] A.-C. Wong and C.-C. Nguyen, "Micromechanical mixer-filters (" mixlers")," J. Microelectromech S, vol. 13, pp. 100-112, 2004.

[25] G. K. Fedder, "CMOS-MEMS resonant mixer-filters," Int. EL Device Meet, (2005), pp. 274-277.

[26] F. Chen, J. Brotz, U. Arslan, C.-C. Lo, T. Mukherjee, and G. K. Fedder, (2005) "CMOS-MEMS resonant RF mixer-filters," in IEEE Int Micro Electro Mech Syst, pp. 24-27.

[27] M. Koskenvuori and I. Tittonen. "GHz-range FSK-reception with microelectromechanical resonators". Sens. Actuators, A, 142(1):346-351, 2008.

[28] AT-H. Lin, JE-Y. Lee, J. Yan, and A.A. Seshia. 'Methods for enhanced electrical transduction and characterization of micromechanical resonators". Sens. Actuators, A,, 158(2):263- 272, 2010.

[29] V. Gouttenoire, T. Barois, S. Perisanu, J.-L. Leclercq, S. T. Purcell, P. Vincent, and A. Ayari. 'Digital and FM demodulation of a doubly clamped single-walled carbon-nanotube oscillator: Towards a nanotube cell phone'. Small, 6(9):1060-1065, 2010.

[30] V. Sazonova, Y. Yaish, H. Ustunel, D. Roundy, T. A. Arias, and P. L. McEuen. 'A tunable carbon nanotube electromechanical oscillator'. Nature, 431(7006):284-287, 2004.

[31] B. Witkamp, M. Poot, and H. S. J. van der Zant. 'Bending-mode vibration of a suspended nanotube resonator'. Nano Lett., 6(12):2904-2908, 2006.

[32] L. J. Hornbeck, "Current status of the digital micromirror device (DMD) for projection television applications," Int. EL Device Meet (1993). pp. 381-384.

[33] D. Dudley, W. M. Duncan, and J. Slaughter, "Emerging digital micromirror device (DMD) applications J. Micromech Microeng, pp. 14-25. 2003.

[34] C.-Y. Tsai and T.-L. Chen, "Design, fabrication and calibration of a novel MEMS logic gate," J. Micromech Microeng, vol. 20, p. 095021, 2010.

[35] C.-Y. Tsai, W.-T. Kuo, C.-B. Lin, and T.-L. Chen, "Design and fabrication of MEMS logic gates," J. Micromech Microeng, vol. 18, p. 045001, 2008.

[36] M. I. Younis, MEMS Linear and Nonlinear Statics and Dynamics: vol. 20: Springer, 2011.

[37] Timoshenko S P and Goodier J N Theory of Elasticity. McGraw-Hill, NewYork, 1970.

[38] A. H. Nayfeh, Introduction to perturbation techniques: John Wiley \& Sons, 2011.

[39] A. Elnagar and A. El-Bassiouny, "Response of self-excited three-degree-of-freedom systems to multifrequency excitations," Int. J. Theor. Phys, vol. 31, pp. 1531-1548, 1992.

[40] ANSYS ${ }^{\circledR}$ Academic Research, Release 15.0, Help system, Structural analysis guide, ANSYS, Inc.

[41] A. Ramini, M. I. Younis, and Q. T. Su, "A low-g electrostatically actuated resonant switch," Smart Mat. Struc, vol. 22, p. 025006, 2013.

[42] J. C. Butcher, The numerical analysis of ordinary differential equations: Runge-Kutta and general linear methods: Wiley-Interscience, 1987.

[43] C. Acar, A, Shkel, MEMS vibratory gyroscopes.Springer, 2008. 